\title{
Colloform high-purity platinum from the placer deposit of Koura River (Gornaya Shoriya, Russia)
}

Nesterenko G.V. ${ }^{1}$, Zhmodik S.M. ${ }^{1,2}$, Airiyants E.V. ${ }^{1}$, Belyanin D.K. ${ }^{1,2}$, Kolpakov V.V. ${ }^{1}$, Bogush

$$
\text { A.A. }{ }^{1,3 *}
$$

${ }^{1}$ V.S. Sobolev Institute of Geology and Mineralogy, Siberian Branch of the Russian Academy of Sciences, Novosibirsk 630090, Russia

${ }^{2}$ Novosibirsk State University, Novosibirsk 630090, Russia

${ }^{3}$ Department of Civil, Environmental \& Geomatic Engineering, University College London, London WC1E 6BT, UK

*Corresponding author: A.A. Bogush, E-mail: annakhol@ gmail.com

\begin{abstract}
A microinclusion of colloform high-purity platinum in a grain of platinum-group minerals (PGM) from the alluvial gold-bearing placer deposit in the south of Western Siberia (Russia) was detected and characterized for the first time. It is different in composition, texture, and conditions of formation from high-purity platinum of other regions described in the literature. The main characteristics of investigated high-purity platinum are colloform-layered texture, admixture of Fe (0.37-0.78 wt.\%), and paragenesis of $\mathrm{Cu}$-rich isoferroplatinum, hongshiite, and rhodarsenide. The PGM grain with high-purity platinum is multiphase and heterogeneous in texture. It is a product of intensive metasomatic transformation of $\mathrm{Cu}$-rich isoferroplatinum $\left(\mathrm{Pt}_{3}\left(\mathrm{Fe}_{0.6} \mathrm{Cu}_{0.4}\right)\right)$. The transformation was carried out in two stages: 1 - copper stage including three substages $(\mathrm{Cu}$-rich isoferroplatinum, copper platinum and hongshiite); and 2 - arsenic (rhodarsenide). The formation of high-purity platinum was separated in time from the formation of isoferroplatinum and was carried out by precipitation from postmagmatic solutions.
\end{abstract}

Keywords: platinum-group elements (PGE), platinum-group minerals (PGM), high-purity platinum, Cu-rich isoferroplatinum, hongshiite, alluvial placer, Gornaya Shoriya

\section{Introduction}

High-purity platinum was discovered in the gold-bearing placer deposit of Koura River situated in Gornaya Shoriya (south-western Siberia, Russia, Fig. 1). This area is in the southern part of the Altai-Sayan orogen formed during several Paleozoic phases of tectonism (Zhmodik et al., 2016). The Lower Cambrian volcanogenic-sedimentary complexes are widely distributed at this site. An important feature of its geological structure is the Seglebirsky ophiolite massif which includes 
amphibolites, gabbro, mafic dikes, and serpentinites (Gusev et al., 2004). PGE mineralization (RuIr-Os alloys) was identified in serpentinites (Gusev et al., 2004).

This is the only grain containing high-purity platinum among the hundreds of mineral grains of platinum group elements (PGE) investigated in the mountain region including Gornaya Shoria, Kuznetsk Alatau and Salair Ridge (Krivenko et al., 1994; Agafonov et al., 1996; Tolstykh et al., 1996 and 1999; Podlipsky and Krivenko, 2001; Zhmodik et al., 2004; Podlipsky et al., 2007). The development of modern high-resolution electron microscopy allows to investigate a material of few nanometers in size which was previously impossible. High-purity platinum is unique and remarkably different from those previously described for the eastern regions of Brazil (Fleet et al., 2002; Cabral et al., 2006), Western Sayan (Tolstykh and Krivenko, 1998), and several regions of the Sakha (Yakutia) Republic (Evstigneeva et al., 1990). The findings of high-purity platinum were mentioned worldwide in other studies (Kingston and El-Dosuky, 1982; Vuorelaien and Tornroos, 1986; Agafonov et al., 1992 and 2000; Rudashevsky et al., 1995; Cabri et al., 1996; Gornostayev et al., 1999; Okrugin, 2000; Angeli, 2005; Chernyshev, 2009; Tolstykh et al., 2002 and 2015; Zaykov et al., 2017).

A goal of this study was to investigate high-purity platinum of unusual morphological type with a detailed discussion of its composition, textural features, and origin.

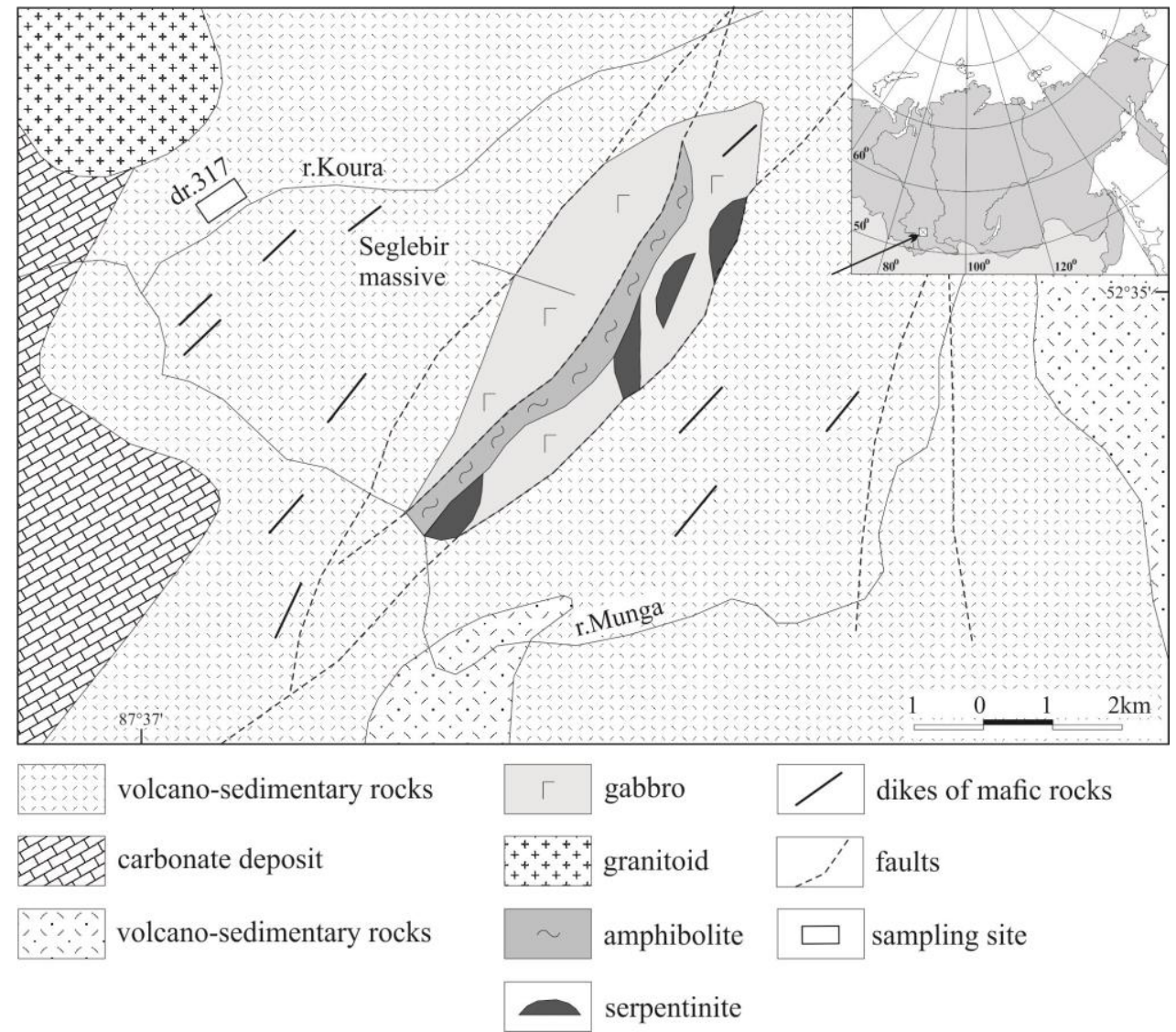

Fig. 1. Geological map of the investigated region showing the location of high-purity platinum at the Seglebirsky massif in Gornaya Shoriya (modified from Gusev et al. (2004)). 


\section{Materials and Methods}

The PGM grain was derived from the heavy mineral concentrate obtained from the No. 317 dredge of the gold-bearing placer deposit of Koura River. The PGM grain was handpicked from the concentrate under a binocular and then examined for grain size, morphology, and surface texture. The PGM grain was mounted to epoxy block and polished with diamond paste for further analysis. The composition and morphology of the sample were investigated at the Analytical Center for Multi-elemental and Isotope Research of the Siberian Branch, Russian Academy of Sciences using a scanning electron microscope (MIRA 3 LMU, Tescan Orsay Holding) equipped with a microanalysis system INCA Energy 450+ X-Max 80 (Oxford Instruments Nanoanalysis Ltd). The analytical conditions were: $20 \mathrm{kV}$ of accelerating voltage with a beam current of $1.6 \mathrm{nA}$ and $10 \mathrm{sec}$ counting time for each analytical spectrum; energy resolution of X-ray spectrometer - 126-127 eV at the $\mathrm{Mn} \mathrm{K}_{\alpha}$ line; and spot size was $12 \mathrm{~nm}$. The spot size of the X-ray beam varies between 5 and 20 microns. Determination of microinclusions with size less than 20 microns was carried out taking into account the composition of the surrounding minerals. The detection limit for most elements was $0.2-0.3 \mathrm{wt} . \%$. The counting time was increased up to 150 seconds in order to decrease detection limit for accurate trace element analysis. More information about parameters of SEM/EDS analysis used in this study can be found in previous work (Zhmodik et al., 2016).

The composition of the PGM grain was also determined with a JEOL JXA-8100 electron microprobe analyser (EMPA) outfitted with a wavelength-dispersive spectrometer (WDS) offering excellent peak separation and accurate trace element analysis. The analytical conditions were accelerating voltage $20 \mathrm{kV}, 100 \mathrm{nA}$ beam current, and $10 \mathrm{sec}$ counting time. Table 1 shows the analytical characteristics of the WDS for determination of the PGM composition. The following standards were used: $\mathrm{Pt}$, Ir, Os, $\mathrm{Pd}$, Rh, and $\mathrm{Ru}$ metals; $\mathrm{CuFeS}_{2}$ for $\mathrm{Cu}, \mathrm{Fe}$, and $\mathrm{S} ; \mathrm{NiFe}_{2} \mathrm{O}_{4}$ for $\mathrm{Ni}$; $\mathrm{FeAsS}$ for $\mathrm{As} ; \mathrm{Sb}_{2} \mathrm{~S}_{3}$ for $\mathrm{Sb}$ (Table 1). Element interference was corrected using experimentally measured coefficients (Lavrent'ev and Usova, 1994). Detection limits of elements were in the range of 0.01-0.02 wt.\%.

\section{Table 1}

Analytical characteristics of WDS for determination of the PGM composition.

\begin{tabular}{|c|c|c|c|c|c|}
\hline Analytical line & $\begin{array}{l}\text { Crystal- } \\
\text { analyzer }\end{array}$ & Standard & Overlapping lines & $\begin{array}{c}\text { Background- } \\
\text { Mm* }\end{array}$ & $\begin{array}{c}\text { Background+ } \\
\text { Mm* }^{*}\end{array}$ \\
\hline $\mathrm{S} \mathrm{K} \alpha$ & PET & $\mathrm{CuFeS}_{2}$ & Pt M3-N4 & 2.0 & 2.0 \\
\hline $\mathrm{Fe} \mathrm{K} \alpha$ & $\mathrm{LiF}$ & $\mathrm{CuFeS}_{2}$ & $\begin{array}{l}\text { Ir L } \gamma 2 \text { II, } \\
\text { Ir L } \gamma 6 \text { II, } \\
\text { Ir L2-O3 II, } \\
\text { Ir L2-O3 II }\end{array}$ & 2.0 & 2.15 \\
\hline $\mathrm{Ni} K \alpha$ & $\mathrm{LiF}$ & $\mathrm{NiFe}_{2} \mathrm{O}_{4}$ & - & 1.5 & 1.5 \\
\hline
\end{tabular}




\begin{tabular}{|c|c|c|c|c|c|}
\hline $\mathrm{Cu} \mathrm{K \alpha}$ & $\mathrm{LiF}$ & $\mathrm{CuFeS}_{2}$ & Ir Lt & 1.3 & 1.3 \\
\hline As L $\alpha$ & TAP & $\mathrm{FeAsS}$ & $\begin{array}{l}\text { Ru L } \alpha 1,2 \text { II, } \\
\text { Sb L } \beta 1 \text { III }\end{array}$ & 2.0 & 2.0 \\
\hline $\mathrm{Ru} \mathrm{L} \alpha$ & PET & Ru-met & $\begin{array}{l}\text { Ir M3-O4, } \\
\text { Pt M3-O1 }\end{array}$ & 3.7 & 1.7 \\
\hline $\mathrm{Rh} L \alpha$ & PET & Rh-met & $\begin{array}{c}\text { Pt M2-N4, } \\
\text { Os Lt III, } \\
\text { Ru L } \beta 1, \\
\text { Cu K } \alpha 1 \text { III }\end{array}$ & 1.25 & 4.8 \\
\hline $\operatorname{Pd} \operatorname{L} \alpha$ & PET & Pd-met & $\begin{array}{l}\text { Pt Lt III, } \\
\text { Ru L } \beta 2, \\
\text { Rh L } \beta 1\end{array}$ & 4.5 & 2.5 \\
\hline $\mathrm{Sb} \mathrm{L} \alpha$ & PET & $\mathrm{Sb}_{2} \mathrm{~S}_{3}$ & 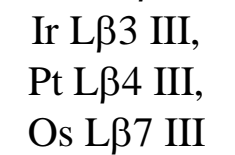 & 1.5 & 2.0 \\
\hline Os $L \beta 1$ & $\mathrm{LiF}$ & Os-met & $\begin{array}{l}\text { Ir L } \beta 17 \text {, } \\
\text { Ir L1-M1 }\end{array}$ & 1.56 & 1.3 \\
\hline $\operatorname{Ir} L \alpha$ & $\mathrm{LiF}$ & Ir-met & 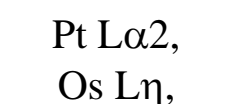 & 0.8 & 1.4 \\
\hline Pt L $\alpha$ & $\mathrm{LiF}$ & Pt-met & 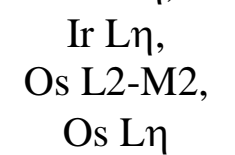 & 2.5 & 1.7 \\
\hline
\end{tabular}

Note: *- Displacement in position of spectrometer relating to the peak

\section{Results}

High-purity platinum was detected in the $0.8 \times 0.6 \mathrm{~mm}$ PGM grain, which has an elongatedlumpy shape with a flattened protrusion (Fig. 2). The surface of the grain is fine-hummocky, finetoothed, and smooth. The fine-toothed surface is characteristic for the most surface of the grain including the gulf-slopes. The oval ledges on the surface of the grain are smooth because of the mechanical river abrasion. The internal structure of the grain is heterogeneous (Figures 2 and 3). There are textures formed both in the magmatic and postmagmatic stages.

The dispersed disseminated nodular (relic) microtexture is common for the PGM grain due to the development of nodules in a heterogeneous matrix. The oval and round forms of inclusions indicate to the occurrence of these textures in the genetic group of textures of liquation differentiation of the melt (Betekhtin et al., 1958). The following microtextures were identified in the matrix: spotted, vein, emulsion, and solid solution decomposition lattice. All of them, with the exception of the last one, are mainly included in the genetic group of textures of hydrothermal(?) substitution. An exception is also the texture of microcracks (Figs 2 and 3 b, d) which is a sign of "fragile deformations" (Betekhtin et al., 1958). 


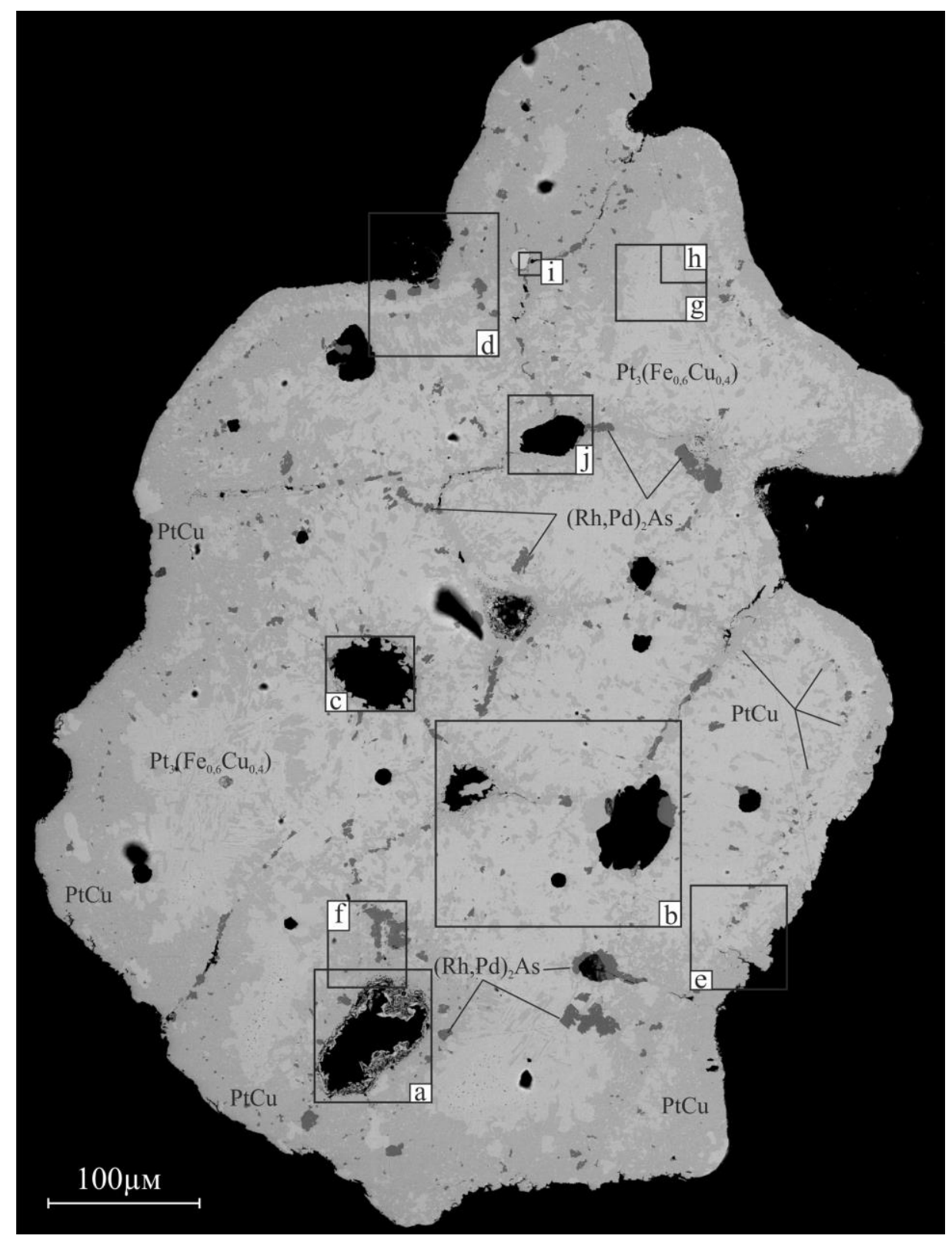

Fig. 2. The texture of the multiphase PGM grain and the position of high-purity platinum (contour a) in it. Light gray - solid solution of $\mathrm{Pt}_{3}\left(\mathrm{Fe}_{0.6} \mathrm{Cu}_{0.4}\right)$ - $\mathrm{Cu}$-rich isoferroplatinum; gray - hongshiite $(\mathrm{PtCu})$; dark gray - rhodarsenide $(\mathrm{Rh}, \mathrm{Pd})_{2} \mathrm{As}$. Rectangular contours are the detailed sections shown in Figure 3. SEM images here and further are in back-scattered electrons.

The main mineral phases of the matrix are solid solutions of platinum and copper as well as the solid solution of $\mathrm{Pt}_{3}\left(\mathrm{Fe}_{0.6} \mathrm{Cu}_{0.4}\right)$ which can be called as $\mathrm{Cu}$-rich isoferroplatinum based on the proximity of its stoichiometric composition to isoferroplatinum. Composition of minerals in the Pt$\mathrm{Cu}-\mathrm{Fe}$ system was described previously (Tolstykh et al., 1996), where authors noted that peripheral parts of $\mathrm{Pt}(\mathrm{Fe})$ grains were usually enriched in copper. $\mathrm{Cu}$-rich isoferroplatinum reveals a stable composition (Table 2, Fig. 4A). The variations in composition relative to the average values is very small: 89.5-90.4 wt.\% (average - 90.1 wt.\%) for Pt (+ Ir, Os, Rh, Ru); 5.2-5.7 wt.\% Fe (5.5 wt.\%); 
3.9-5.1 wt.\% $\mathrm{Cu}$ (4.2 wt.\%). Cu-rich isoferroplatinum contains decomposition products of solid solution which are represented by the lamellas of $\mathrm{Os}_{0.4} \mathrm{Ir}_{0.4} \mathrm{Ru}_{0.2}$ intermetallic compound (Fig. $3 \mathrm{f}$, h). According to the phase diagrams obtained during the investigation of the Pt-Os system (Winkler, 1943; Voronova et al., 1984), two regions of solid solutions were identified: 1) Os with Pt (about 11 at.\% Pt); 2) Pt with Os (up to 25 at.\% Os). The total concentration of osmium in the studied grain does not exceed 5 at.\%. According to this two-component diagram of Pt-Os and osmium content, osmium is not able to deposit from this system. However, a solid solution of platinum and osmium is formed. Additionally, it should be noted that in our case the system is multicomponent consisting of Pt, Os, Fe, Ir, and Ru. Based on our data, we concluded that inclusions of Os-Ir-Ru alloys in isoferroplatinum are decomposition products of multicomponent solid solution. It should be noted that in our case we are talking about rutheniridosmine $\left(\mathrm{Os}_{0.4} \operatorname{Ir}_{0.4} \mathrm{Ru}_{0.2}\right)$, with the composition similar to ruthenosmiridium $\left(\mathrm{Ir}_{0.4} \mathrm{Os}_{0.4} \mathrm{Ru}_{0.2}\right)$. The host matrix of $\mathrm{Cu}$-rich isoferroplatinum (Table 3) has a significant effect on the lamella composition due to their ultra-small size. The total amount of RuOs-Ir intermetallic compound in the PGM grain is several wt.\%.

\section{Table 2}

Composition of $\mathrm{Cu}$-rich isoferroplatinum according to SEM/EDS (1-17) and WDS (18-22), wt.\%.

\begin{tabular}{ccccccccc}
\hline $\begin{array}{c}\text { Anal. } \\
\text { No. }\end{array}$ & $\mathrm{Fe}$ & $\mathrm{Cu}$ & $\mathrm{Ru}$ & $\mathrm{Os}$ & $\mathrm{Ir}$ & $\mathrm{Pt}$ & Totals & Figure \\
\hline 1 & 5.62 & 4.75 & 0.56 & & & 89.95 & 100.89 & $3 \mathrm{II}$ \\
2 & 5.59 & 3.57 & & & & 89.88 & 99.04 & $3 \mathrm{~b}$ \\
3 & 5.17 & 3.48 & 0.74 & 1.58 & 2.54 & 85.38 & 98.88 & \\
\hline 4 & 5.33 & 4.28 & & & & 88.47 & 98.08 & \\
5 & 5.07 & 3.79 & 0.82 & 2.85 & 2.61 & 83.02 & 98.16 & $3 \mathrm{c}$ \\
6 & 5.11 & 3.72 & 0.5 & 2.65 & 3.52 & 85.91 & 101.43 & \\
\hline 7 & 4.88 & 3.64 & 0.73 & 2.07 & 3.99 & 84.99 & 100.29 & \\
8 & 5.52 & 3.84 & 0.51 & & & 87.96 & 97.84 & $3 \mathrm{~d}$ \\
9 & 5.45 & 4.34 & & & & 89.71 & 99.51 & \\
\hline 10 & 5.28 & 3.7 & & & 1.9 & 87.95 & 98.82 & \multirow{2}{3}{$\mathrm{e}$} \\
11 & 5.6 & 3.98 & 0.48 & & & 91.93 & 101.99 & \\
\hline 12 & 5.07 & 3.62 & 0.58 & 1.94 & 2.61 & 86.67 & 100.49 & \\
13 & 5.12 & 3.84 & 0.49 & 2.22 & 2.36 & 86.61 & 100.64 & $3 \mathrm{~g}$ \\
14 & 4.82 & 4.01 & 0.54 & 1.57 & 1.52 & 86.46 & 98.92 & \\
\hline 15 & 4.63 & 3.7 & 1.56 & 7.97 & 6.98 & 74.11 & 98.95 & \\
16 & 5.16 & 4.42 & & & & 87.53 & 97.11 & $3 \mathrm{j}$ \\
17 & 5.24 & 4.27 & & & & 87.23 & 96.75 & \\
\hline 18 & 5.41 & 3.98 & 0.28 & 0.20 & 0.70 & 88.68 & 99.25 & \\
19 & 5.15 & 4.02 & 0.58 & 2.47 & 2.33 & 84.42 & 98.97 & \\
20 & 5.39 & 3.77 & 0.34 & 1.41 & 1.44 & 86.66 & 99.01 & 2 \\
21 & 5.45 & 3.74 & 0.19 & 0.93 & 1.39 & 87.65 & 99.35 & \\
22 & 5.57 & 3.83 & 0.10 & & 0.25 & 89.55 & 99.30 & \\
\hline & & & & & & & & \\
\hline
\end{tabular}




\section{Table 3}

The composition of Ru-Os-Ir lamellas included in the $\mathrm{Cu}$-rich isoferroplatinum and $\mathrm{Cu}-\mathrm{Pt}$ alloy using SEM/EDS, wt.\%.

\begin{tabular}{|c|c|c|c|c|c|c|c|c|c|c|c|c|}
\hline $\begin{array}{c}\text { Anal. } \\
\text { No. }\end{array}$ & $\mathrm{Fe}$ & $\mathrm{Cu}$ & As & $\mathrm{Ru}$ & $\mathrm{Rh}$ & $\mathrm{Pd}$ & Os & Ir & $\mathrm{Pt}$ & Total & $\mathrm{Zm} *$ & Figure \\
\hline 1 & & 15.8 & & 3.0 & & & 12.8 & 10.2 & 56.6 & 98.4 & 2 & \multirow{2}{*}{$3 \mathrm{a}$} \\
\hline 2 & 4.48 & 3.6 & & 2.64 & & & 9.55 & 10.39 & 70.7 & 101.37 & 1 & \\
\hline 3 & 4.07 & 4.26 & & 2.78 & & & 11.21 & 9.8 & 65.69 & 97.8 & 1 & $3 \mathrm{j}$ \\
\hline 4 & \multirow[t]{2}{*}{3.0} & 5.7 & & 2.2 & & & 12.3 & 10.1 & 66.7 & 99.9 & 1 & \multirow{2}{*}{$3 f$} \\
\hline 5 & & 13.2 & & 1.7 & & & 6.1 & 6.3 & 73.0 & 100.3 & 2 & \\
\hline 6 & \multirow{3}{*}{0.3} & 14.3 & & 1.9 & & & 6.6 & 7.6 & 70.1 & 100.5 & 2 & \multirow{5}{*}{$3 \mathrm{~h}$} \\
\hline 7 & & 10.3 & & 4.1 & & & 21.0 & 16.7 & 48.9 & 101.3 & 2 & \\
\hline 8 & & 8.8 & & 3.1 & & & 13.7 & 12.7 & 62.3 & 100.6 & 2 & \\
\hline 9 & 0.2 & 9.1 & & 3.5 & & & 13.8 & 12.4 & 61.3 & 100.2 & 2 & \\
\hline 10 & & 8.8 & & 3.2 & & & 14.1 & 11.1 & 62.5 & 99.7 & 2 & \\
\hline
\end{tabular}

Note: $* \mathrm{Zm}-$ enclosing minerals: $1-\mathrm{Cu}$-rich isoferroplatinum; $2-\mathrm{Cu}-\mathrm{Pt}$ alloy.

Two individual mineral phases are distinguished in solid solutions of platinum and copper: hongshiite and a $\mathrm{Cu}-\mathrm{Pt}$ intermetallic compound. Hongshiite differs from its stoichiometric composition due to deficiency of copper: $\mathrm{Pt}_{1.2} \mathrm{Cu}_{0.8}-\mathrm{Pt}_{1.1} \mathrm{Cu}_{0.9}$ (Table 4, Fig. 4A) with an average copper content of $19.14 \mathrm{wt} \%$. Cu-Pt intermetallic compound is characterized by an even greater copper deficiency - $\mathrm{Pt}_{1.4} \mathrm{Cu}_{0.8}(\mathrm{Cu}$ average content - 11.7 wt.\%) (Table 5, Fig. 4A). Lamellas of $\mathrm{Ru}-$ Os-Ir intermetallic compound were identified (Table 3 and Fig. 3 f, h) in $\mathrm{Cu}-\mathrm{Pt}$ intermetallic compound and $\mathrm{Cu}$-rich isoferroplatinum in same amount and composition - $\mathrm{Os}_{0.4} \mathrm{Ir}_{0.4} \mathrm{Ru}_{0.2}$ or $\mathrm{Ir}_{0.4} \mathrm{Os}_{0.4} \mathrm{Ru}_{0.2}$ (rutheniridosmine or ruthenosmiridium). These lamellas in $\mathrm{Cu}-\mathrm{Pt}$ intermetallic compound are probably relicts inherited from $\mathrm{Cu}$-rich isoferroplatinum. Whereas, the lamellas in $\mathrm{Cu}$-rich isoferroplatinum are relicts inherited from $\mathrm{Pt}-\mathrm{Fe}$ alloy (isoferroplatinum). $\mathrm{Cu}-\mathrm{Pt}$ intermetallic compound and $\mathrm{Cu}$-rich isoferroplatinum closely associate with each other forming a spotted texture with predominance of one of these minerals in the different parts of the PGM grain (Figs 2 and 3 II, d, f, j). Hongshiite is mainly at the marginal zone of the grain, forming an unclear texture (Figs 2 and 3 e).

\section{Table 4}

Hongshiite composition based on SEM/EDS (points 1-23) and WDS (points 24-29), wt.\%.

\begin{tabular}{|c|c|c|c|c|c|c|c|c|}
\hline $\begin{array}{c}\text { Anal. } \\
\text { No. }\end{array}$ & $\mathrm{Fe}$ & $\mathrm{Cu}$ & $\mathrm{Ru}$ & Os & $\mathrm{Ir}$ & $\mathrm{Pt}$ & Totals & Figure \\
\hline 1 & & 20.0 & 0.6 & 1.3 & & 76.9 & 98.7 & $3 a$ \\
\hline 2 & & 19.3 & & & & 78.8 & 98.1 & \\
\hline 3 & & 18.8 & & & & 78.6 & 97.4 & \\
\hline 4 & & 18.1 & 0.4 & 1.6 & 1.5 & 77.7 & 99.4 & $3 \mathrm{~b}$ \\
\hline
\end{tabular}




\begin{tabular}{|c|c|c|c|c|c|c|c|c|}
\hline 5 & 0.6 & 18.5 & & & & 77.3 & 96.5 & \\
\hline 6 & & 18.8 & & & & 78.0 & 96.8 & \\
\hline 7 & & 18.8 & & & 1.9 & 80.1 & 100.8 & \multirow{6}{*}{$3 \mathrm{c}$} \\
\hline 8 & 0.2 & 20.1 & & & & 77.5 & 97.8 & \\
\hline 9 & 0.3 & 19.0 & & & & 78.5 & 97.7 & \\
\hline 10 & 0.2 & 19.1 & 0.5 & & & 76.1 & 96.0 & \\
\hline 11 & 0.3 & 19.6 & & & & 76.7 & 96.5 & \\
\hline 12 & 0.4 & 20.0 & & & & 77.2 & 97.5 & \\
\hline 13 & & 19.2 & & & & 80.6 & 99.8 & $3 \mathrm{~d}$ \\
\hline 14 & & 19.0 & & & & 80.2 & 99.1 & $3 \mathrm{e}$ \\
\hline 15 & & 20.8 & & & & 76.0 & 96.8 & \multirow{4}{*}{$3 \mathrm{f}$} \\
\hline 16 & & 19.8 & & & & 77.3 & 97.1 & \\
\hline 17 & & 18.2 & & & & 79.4 & 97.5 & \\
\hline 18 & & 19.9 & & & & 77.2 & 97.0 & \\
\hline 19 & & 20 & & & & 79.5 & 99.4 & $3 \mathrm{~g}$ \\
\hline 20 & & 19.5 & 0.3 & 1.0 & & 78.3 & 99.1 & $3 \mathrm{~h}$ \\
\hline 21 & & 20.0 & & 2.2 & 1.6 & 76.0 & 99.9 & \multirow{3}{*}{$3 \mathrm{i}$} \\
\hline 22 & 0.3 & 20.2 & & & & 76.6 & 97.1 & \\
\hline 23 & 0.7 & 18.1 & 0.4 & & & 76.6 & 95.9 & \\
\hline 24 & 0.05 & 18.75 & 0.65 & 2.21 & 2.04 & 74.42 & 98.12 & \multirow{6}{*}{2} \\
\hline 25 & 0.04 & 19.59 & 0.27 & 1.37 & 1.27 & 75.17 & 97.71 & \\
\hline 26 & 0.1 & 18.62 & 0.04 & 0.59 & 0.35 & 79.0 & 98.7 & \\
\hline 27 & 0.1 & 18.98 & 0.32 & 1.28 & 0.85 & 76.82 & 98.34 & \\
\hline 28 & 0.08 & 16.34 & 0.2 & 1.86 & 2.04 & 77.85 & 98.36 & \\
\hline 29 & 0.27 & 17.95 & 0.62 & 2.72 & 2.13 & 74.95 & 98.64 & \\
\hline
\end{tabular}

Table 5

Composition of $\mathrm{Cu}-\mathrm{Pt}$ intermetallic compound based on SEM/EDS, wt.\%.

\begin{tabular}{ccccccccc}
\hline $\begin{array}{c}\text { Anal. } \\
\text { No. }\end{array}$ & $\mathrm{Fe}$ & $\mathrm{Cu}$ & $\mathrm{Ru}$ & $\mathrm{Os}$ & $\mathrm{Ir}$ & $\mathrm{Pt}$ & Totals & Figure \\
\hline 1 & & 11.5 & 0.8 & 2.0 & 1.6 & 84.3 & 100.2 & $3 \mathrm{a}$ \\
\hline 2 & 0.3 & 11.1 & 0.7 & 2.6 & 2.6 & 83.2 & 100.5 & $3 \mathrm{c}$ \\
\hline 3 & & 12.3 & & & & 87.4 & 99.6 & $3 \mathrm{f}$ \\
\hline 4 & & 11.6 & 0.3 & 1.9 & 2.1 & 85.2 & 101.0 & $3 \mathrm{~g}$ \\
5 & & 12.1 & & & & 87.1 & 99.2 & \\
\hline 6 & & 11.7 & 0.8 & 3.0 & 2.7 & 82.5 & 100.7 & $3 \mathrm{~h}$ \\
7 & 0.2 & 11.4 & 0.5 & 1.8 & 2.6 & 84.1 & 100.6 & \\
\hline
\end{tabular}

The inclusions of arsenide and sulfoarsenide minerals were identified in the grain: rhodarsenide, irarsite, sperrylite, and platarsite (Figs 3c, i, j). The most widely distributed mineral is rhodarsenide which forms an euhedral crystal of less than 4-6 $\mu \mathrm{m}$ in the solid solution of platinum and copper. These phases form either chains on the periphery of the grain (Fig. 3d) or their intergrowths of the panidiomorphic-granular structure forming clusters and veins confined to microcracks, intersecting the host grain in different directions (Figs 2 and 3 b, f, i). Microemulsion impregnation of rhodarsenide in $\mathrm{Cu}$-rich isoferroplatinum was also observed (Fig. 3g). The rhodium and palladium 
were detected in rhodarsenide (Table 6, Fig. 4b) two groups: $\left(\operatorname{RhPd}_{0.8} \mathrm{Pt}_{0.2}\right)_{2} \mathrm{As}$ and $\left(\mathrm{Rh}_{1.4} \mathrm{Pd}_{0.3} \mathrm{Pt}_{0.2}\right)_{1.9} \mathrm{As}_{1.1}$.
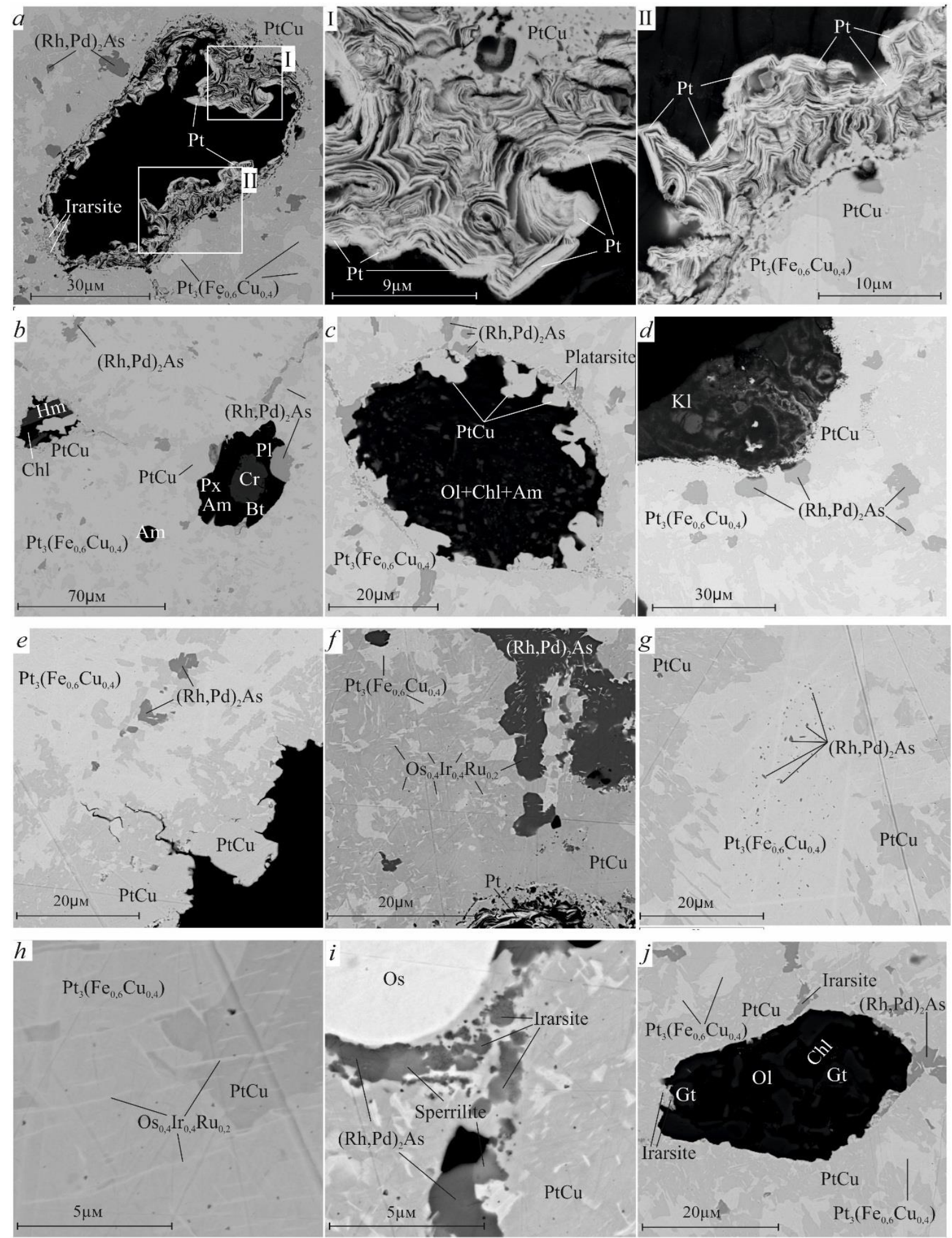

Fig 3. Internal textures defined in figure 2.

$a$ - high-purity platinum in the inclusions; I, II - enlarged micro-areas allocated to a; $b, c, j-$ inclusions filled with silicates and oxides; $d$ - a notch on the grain surface filled with kaolinitecontaining material; $; e$-hongshiite in the marginal part of the grain;; $f$-inclusion of rhodarsenide 
with Os-Ir-Ru lamellas; $g$ - emulsion inclusions of rhodarsenide; $h$ - Os-Ir-Ru lamellas penetrated through $\mathrm{Cu}$-rich isoferroplatinum and hongshiite; $i$ - round inclusion of osmium. Mineral abbreviation: Am - amphibole, $\mathrm{Bt}$ - biotite, $\mathrm{Chl}$ - chlorite, $\mathrm{Cr}$ - chromite, $\mathrm{Gt}$ - goethite, $\mathrm{Hm}$ hematite, $\mathrm{Kl}$ - kaolinite, $\mathrm{Ol}$ - olivine, $\mathrm{Pl}$ - plagioclase, $\mathrm{Px}$ - pyroxenite.

\section{Table 6}

Rhodarsenide composition based on SEM/EDS (points 1-18) and WDS (points 19-25), wt.\%.

\begin{tabular}{|c|c|c|c|c|c|c|c|c|c|c|c|c|}
\hline $\begin{array}{l}\text { Anal. } \\
\text { No. }\end{array}$ & $\mathrm{Fe}$ & $\mathrm{Cu}$ & As & $\mathrm{Ru}$ & $\mathrm{Rh}$ & $P d$ & Os & Ir & $\mathrm{Pt}$ & Total & Figure & Formula composition \\
\hline 1 & & 1.0 & 20.3 & 0.9 & 29.3 & 25.5 & 5.6 & 4.3 & 14.0 & 100.8 & $3 a$ & $\left(\mathrm{Rh}_{0.91} \mathrm{Pd}_{0.76} \mathrm{Pt}_{0.23} \mathrm{Os}_{0.09} \mathrm{Ir}_{0.07} \mathrm{Ru}_{0.03} \mathrm{Cu}_{0.05}\right)_{2.14} \mathrm{As}_{0.86}$ \\
\hline 2 & & & 23.2 & & 33.1 & 28.0 & & & 14.6 & 98.9 & & $\left(\mathrm{Rh}_{1.00} \mathrm{Pd}_{0.81} \mathrm{Pt}_{0.23}\right)_{2.04} \mathrm{As}_{0.96}$ \\
\hline 3 & & & 23.4 & & 33.0 & 28.6 & & & 14.1 & 99.1 & & $\left(\mathrm{Rh}_{0.99} \mathrm{Pd}_{0.83} \mathrm{Pt}_{0.22}\right)_{2.04} \mathrm{As}_{0.96}$ \\
\hline 4 & & & 23.4 & & 33.0 & 29.6 & & & 13.7 & 99.7 & $3 b$ & $\left(\mathrm{Rh}_{0.98} \mathrm{Pd}_{0.85} \mathrm{Pt}_{0.21}\right)_{2.05} \mathrm{As}_{0.95}$ \\
\hline 5 & 0.6 & 0.5 & 26.3 & & 48.2 & 10.0 & & & 15.1 & 100.6 & & $\left(\mathrm{Rh}_{1.39} \mathrm{Pd}_{0.28} \mathrm{Pt}_{0.23} \mathrm{Cu}_{0.02} \mathrm{Fe}_{0.03}\right)_{1.96} \mathrm{As}_{1.04}$ \\
\hline 6 & & 0.3 & 27.3 & & 50.3 & 11.3 & & & 12.6 & 101.8 & & $\left(\mathrm{Rh}_{1.43} \mathrm{Pd}_{0.31} \mathrm{Pt}_{0.19} \mathrm{Cu}_{0.01}\right)_{1.94} \mathrm{As}_{1.06}$ \\
\hline 7 & 0.2 & 1.6 & 22.4 & 0.6 & 31.2 & 27.0 & 2.0 & & 15.9 & 100.8 & & $\left(\mathrm{Rh}_{0.93} \mathrm{Pd}_{0.77} \mathrm{Pt}_{0.25} \mathrm{Cu}_{0.08} \mathrm{Os}_{0.03} \mathrm{Ru}_{0.02} \mathrm{Fe}_{0.01}\right)_{2.09} \mathrm{As}_{0.9}$ \\
\hline 8 & & 0.4 & 24.1 & & 33.4 & 29.4 & & & 14.6 & 101.8 & $3 c$ & $\left(\mathrm{Rh}_{0.97} \mathrm{Pd}_{0.83} \mathrm{Pt}_{0.22} \mathrm{Cu}_{0.02}\right)_{2.04} \mathrm{As}_{0.96}$ \\
\hline 9 & & 0.7 & 23.9 & & 33.8 & 29.3 & & & 14.2 & 101.9 & & $\left(\mathrm{Rh}_{0.98} \mathrm{Pd}_{0.82} \mathrm{Pt}_{0.22} \mathrm{Cu}_{0.03}\right)_{2.05} \mathrm{As}_{0.95}$ \\
\hline 10 & & 2.2 & 22.4 & & 30.4 & 25.6 & & & 19.7 & 100.2 & & $\left(\mathrm{Rh}_{0.91} \mathrm{Pd}_{0.74} \mathrm{Pt}_{0.31} \mathrm{Cu}_{0.11}\right)_{2.08} \mathrm{As}_{0.92}$ \\
\hline 11 & & 0.5 & 23.4 & & 33.1 & 28.6 & & & 15.0 & 100.7 & & $\left(\mathrm{Rh}_{0.98} \mathrm{Pd}_{0.82} \mathrm{Pt}_{0.23} \mathrm{Cu}_{0.02}\right)_{2.05} \mathrm{As}_{0.95}$ \\
\hline 12 & & 0.7 & 23.3 & & 33.7 & 28.4 & & & 15.8 & 102.0 & & $\left(\mathrm{Rh}_{0.98} \mathrm{Pd}_{0.80} \mathrm{Pt}_{0.24} \mathrm{Cu}_{0.03}\right)_{2.06} \mathrm{As}_{0.94}$ \\
\hline 13 & 1.9 & 2.3 & 15.4 & 0.7 & 20.5 & 16.9 & 2.8 & 2.8 & 37.0 & 100.3 & $3 \mathrm{~d}$ & \\
\hline 14 & & 0.78 & 23.2 & & 33.1 & 27.6 & 0.7 & & 15.0 & 100.4 & & $\left(\mathrm{Rh}_{0.98} \mathrm{Pd}_{0.79} \mathrm{Pt}_{0.23} \mathrm{Os}_{0.01} \mathrm{Cu}_{0.04}\right)_{2.06} \mathrm{As}_{0.94}$ \\
\hline 15 & & 4.0 & 21.1 & 2.8 & 24.3 & 20.1 & 6.5 & 6.7 & 14.4 & 100.0 & $3 \mathrm{e}$ & $\left(\mathrm{Rh}_{0.75} \mathrm{Pd}_{0.60} \mathrm{Pt}_{0.24} \mathrm{Os}_{0.11} \mathrm{Ir}_{0.11} \mathrm{Ru}_{0.09} \mathrm{Cu}_{0.02}\right)_{2.10} \mathrm{As}_{0.90}$ \\
\hline 16 & 2.8 & 2.0 & 11.0 & 0.5 & 22.0 & 5.3 & 1.0 & 1.3 & 53.8 & 99.7 & $3 f$ & \\
\hline 17 & 3.1 & 2.3 & 9.1 & 0.7 & 14.2 & 10.8 & & 1.6 & 57.0 & 98.7 & $\mathrm{I}$ & \\
\hline 18 & & 1.6 & 23.2 & & 31.6 & 27.5 & & & 15.8 & 99.7 & $3 \mathrm{i}$ & $\left(\mathrm{Rh}_{0.94} \mathrm{Pd}_{0.79} \mathrm{Pt}_{0.25} \mathrm{Cu}_{0.08}\right)_{2.05} \mathrm{As}_{0.95}$ \\
\hline 19 & 0.01 & 0.44 & 24.9 & & 32.8 & 27.8 & & 0.08 & 14.18 & 100.2 & & $\left(\mathrm{Rh}_{0.96} \mathrm{Pd}_{0.79} \mathrm{Pt}_{0.22} \mathrm{Cu}_{0.02}\right)_{1.99} \mathrm{As}_{1.01}$ \\
\hline 20 & 0.03 & 0.47 & 25.0 & & 32.5 & 27.9 & & & 14.12 & 100.0 & & $\left(\mathrm{Rh}_{0.96} \mathrm{Pd}_{0.79} \mathrm{Pt}_{0.22} \mathrm{Cu}_{0.02}\right)_{1.99} \mathrm{As}_{1.01}$ \\
\hline 21 & 0.02 & 0.49 & 25.0 & & 32.0 & 28.4 & & 0.03 & 14.21 & 100.1 & & $\left(\mathrm{Rh}_{0.94} \mathrm{Pd}_{0.81} \mathrm{Pt}_{0.22} \mathrm{Cu}_{0.02}\right)_{1.99} \mathrm{As}_{1.01}$ \\
\hline 22 & 0.02 & 0.45 & 24.7 & & 32.0 & 28.5 & & 0.01 & 14.28 & 99.98 & 2 & $\left(\mathrm{Rh}_{0.94} \mathrm{Pd}_{0.81} \mathrm{Pt}_{0.22} \mathrm{Cu}_{0.02}\right)_{2.00} \mathrm{As}_{1.00}$ \\
\hline 23 & 0.02 & 0.34 & 24.8 & & 32.1 & 28.6 & & 0.02 & 14.31 & 100.1 & & $\left(\mathrm{Rh}_{0.94} \mathrm{Pd}_{0.81} \mathrm{Pt}_{0.22} \mathrm{Cu}_{0.02}\right)_{2.00} \mathrm{As}_{1.00}$ \\
\hline 24 & 0.06 & 0.41 & 28.8 & & 47.7 & 10.7 & & 0.09 & 12.66 & 100.4 & & $\left(\mathrm{Rh}_{1.36} \mathrm{Pd}_{0.30} \mathrm{Pt}_{0.19} \mathrm{Cu}_{0.02}\right)_{1.87} \mathrm{As}_{1.13}$ \\
\hline 25 & 0.11 & 0.50 & 28.9 & & 47.6 & 10.9 & & 0.38 & 13.38 & 101.8 & & $\left(\mathrm{Rh}_{1.35} \mathrm{Pd}_{0.30} \mathrm{Pt}_{0.20} \mathrm{Cu}_{0.02} \mathrm{Ir}_{0.01} \mathrm{Fe}_{0.01}\right)_{1.88} \mathrm{As}_{1.12}$ \\
\hline
\end{tabular}

Note: Anal. No. 13, 16 and 17 - rhodarsenide-2 (fine emulsion inclusion in $\mathrm{Cu}$-rich isoferroplatinum).

The nodule inclusions are quite diverse in texture and composition (Fig. 2). They have predominantly flattened shapes and ideally rounded ones in the smallest "drops" (Figs 2 and 3b) from $10 \times 10 \mu \mathrm{m}$ to $40 \times 70 \mu \mathrm{m}$ in size. The nodule inclusions are filled entirely or partly with rockforming silicates (Table 7 and Fig. 3b, d), PGM (Fig. 3 a-c), and/or Fe and Cr oxides (Fig. 3b). Most phenocrysts have a polymineral composition (Fig. 3b, c) but some of them are monomineralic, represented, for example, by native osmium (Figs 2 and 3) and amphibole (Fig. 3b, small inclusion). Along with syngenetic minerals of typically magmatic genesis, such as olivine, 
chromite, and native osmium, epigenetic postmagmatic minerals are present in the inclusions: hematite, hongshiite, rhodarsenide, etc. The micro-extractions of these minerals form outgrowths on the walls of nodules (Fig. 3b, c). The composition of hongshiite and rhodarsenide in the main part of the grain and on the walls of the inclusions are similar (Fig. 4a, b). Goethite was observed in one phenocryst (Fig. 3j).

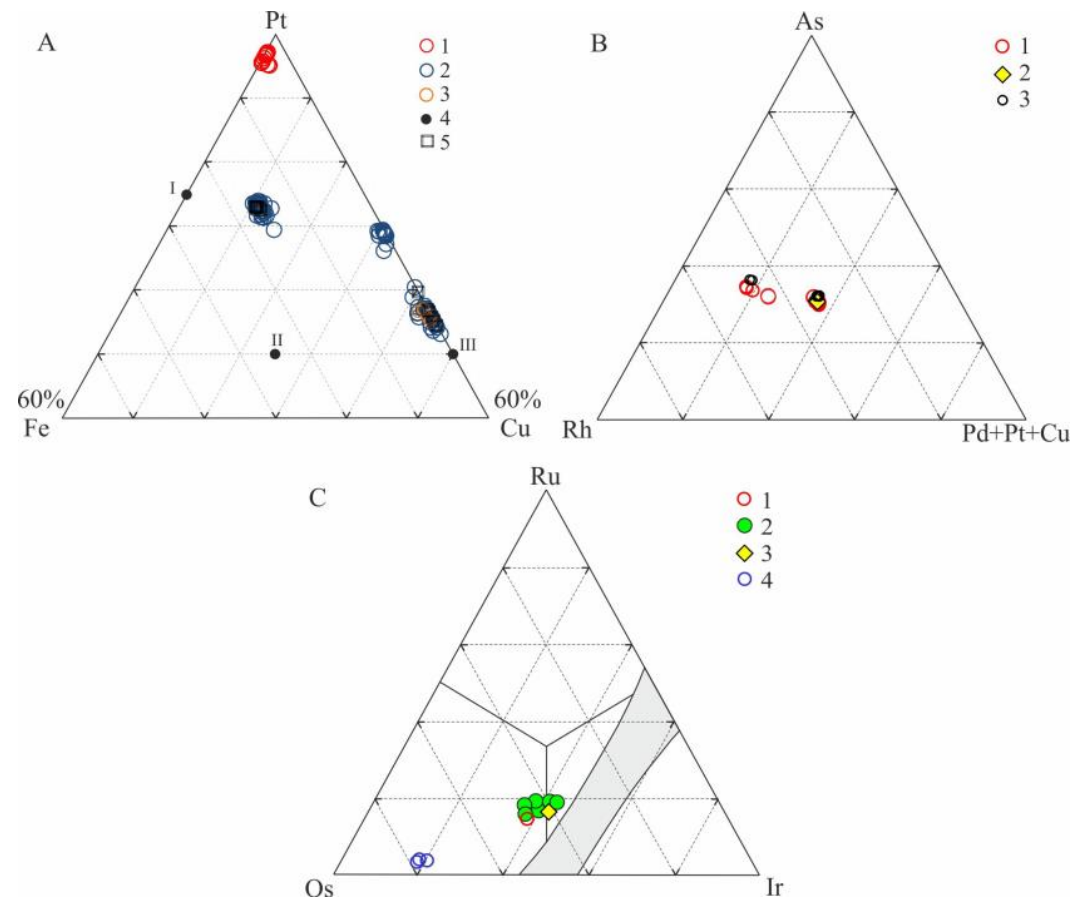

Fig. 4. Triangular diagrams of the PGM compositions based on SEM/EDS and WDS.

A - Fe-Pt-Cu system: 1 - high-purity platinum; 2 - Cu-rich isoferroplatinum, $\mathrm{Cu}-\mathrm{Pt}$ alloy, and hongshiite; 3 - hongshiite (1-3 analysis based on SEM-EDS); 4 - Minerals of stoichiometric composition (I - isoferroplatinum, II - tulameenite, and II - hongshiite); 5 - Cu-rich isoferroplatinum, $\mathrm{Cu}-\mathrm{Pt}$ alloy, and hongshiite based on WDS. B - Rh-As-(Pd+Pt+Cu) system: 1 Idiomorphic inclusions of rhodarsenide in the matrix; 2 - rhodarsenide in the microcavity; 3 rhodarsenide based on WDS. C - Ru-Ir-Os system: 1 - Ru-Ir-Os lamellas in Cu-rich isoferroplatinum; 2 - Ru-Ir-Os lamellas in Cu-Pt alloy; 3 - Ru-Ir-Os lamellas in rhodarsenide; 4 round inclusion of osmium in hongshiite.

\section{Table 7}

Compositions of silicates in nodular textures based on SEM/EDS, wt.\%.

\begin{tabular}{|c|c|c|c|c|c|c|c|c|c|c|c|c|}
\hline $\begin{array}{c}\text { Anal. } \\
\text { No. }\end{array}$ & $\mathrm{O}$ & $\mathrm{Fe}$ & $\mathrm{Na}$ & $\mathrm{Mg}$ & $\mathrm{Al}$ & $\mathrm{Si}$ & $\mathrm{K}$ & $\mathrm{Ca}$ & te & Totals & Mineral & Figure \\
\hline 1 & 43.82 & 7.96 & 0.2 & 9.07 & 2.15 & 22.35 & \multirow{4}{*}{0.39} & 13.57 & 0.16 & 99.28 & Px & \multirow{4}{*}{$3 \mathrm{~b}$} \\
\hline 2 & 46.44 & 0.3 & 6.14 & 1.11 & 11.18 & 27.92 & & 3.45 & & 96.93 & $\mathrm{Pl}$ & \\
\hline 3 & 43.93 & 3.86 & 0.73 & 9.39 & 2.28 & 24.98 & & 13.43 & 0.46 & 99.07 & $\mathrm{Px}$ & \\
\hline 4 & 43.13 & 6.97 & 0.18 & 9.34 & 2.16 & 23.08 & & 14.0 & 1.34 & 99.27 & Px & \\
\hline
\end{tabular}




\begin{tabular}{ccccccccccccc}
5 & 50.48 & 3.1 & & 11.55 & 7.09 & 17.14 & 6.75 & 2.55 & 3.01 & 102.53 & Bt & \\
6 & 43.11 & 6.35 & 0.22 & 9.39 & 1.81 & 23.11 & & 13.8 & 0.34 & 98.13 & Px \\
7 & 45.83 & 6.23 & 1.45 & 9.61 & 6.06 & 20.85 & 0.5 & 8.36 & 2.69 & 101.59 & Am & \\
8 & 43.68 & 8.56 & 0.95 & 7.1 & 3.34 & 22.55 & 0.43 & 11.58 & 1.42 & 99.94 & Px & \\
\hline 9 & 36.88 & 46.83 & 0.26 & 7.35 & 1.52 & 10.16 & 0.65 & 1.81 & 1.78 & 107.24 & Ol \\
10 & 45.58 & 3.58 & 0.89 & 13.07 & 5.76 & 20.79 & 0.51 & 5.66 & 0.71 & 96.55 & Am \\
11 & 44.93 & 4.27 & 0.33 & 13.38 & 4.3 & 21.85 & 1.52 & 4.71 & 1.17 & 96.45 & Am & \\
12 & 45.42 & 4.69 & 0.33 & 12.47 & 1.58 & 25.01 & 0.31 & 7.94 & 0.33 & 98.08 & Am & $3 \mathrm{c}$ \\
13 & 43.12 & 8.77 & 0.38 & 11.51 & 2.37 & 22.09 & 0.52 & 8.08 & 0.83 & 97.66 & Am & \\
14 & 43.52 & 8.33 & 0.35 & 12.39 & 2.66 & 22.39 & 0.62 & 6.56 & 0.67 & 97.49 & Am & \\
15 & 44.46 & 4.47 & 0.19 & 12.94 & 1.28 & 25.0 & 0.27 & 7.77 & 1.37 & 96.93 & Am & \\
16 & 41.91 & 28.26 & 0.31 & 9.32 & 1.72 & 16.56 & 0.36 & 4.78 & 0.93 & 104.15 & Ol & \\
\hline
\end{tabular}

Minerals: $\mathrm{Px}$ - pyroxene, $\mathrm{Bt}$ - biotite, $\mathrm{Pl}$ - plagioclase, $\mathrm{Am}$ - amphibole, $\mathrm{Ol}$ - olivine; te - trace elements - Ti, Mn, Ni, Cr, Pt.

High-purity platinum was found in one microscopic oval cavity, being some $40 \times 80 \mu \mathrm{m}$ in size (Fig. 2). The delicate layer of high-purity platinum with thickness varying from 3-6 to $20 \mu \mathrm{m}$, covers walls of the cavity. The microtexture is colloform-layered, similar to the texture of malachite. The sublayers $(0.15-1.0 \mu \mathrm{m})$ of native platinum are interlayered with the empty and semi-empty sublayers containing oxygen, magnesium, aluminium, and silicon (Fig. 3a, I-II). The layer of hongshiite $(1.0-1.5 \mu \mathrm{m})$ occurs on the wall of the cavity underneath the layer of high-purity platinum (Fig. 3 II). The contact of the layer of high-purity platinum with the wall of the microcavity is sharp and the individual sublayers are perpendicular to the wall. The chemical composition of platinum layer is shown in Table 8. Iron (0.4-0.8 wt.\%) was determined in highpurity Pt (Fig. 4a). Cu was found in $60 \%$ of the analyses, Ir and $\mathrm{Ru}-$ in $20 \%$, and $\mathrm{Rh}-$ in $13 \%$, and Os was below detection limit. Correlation of the content of minor elements with the fineness of platinum was observed only for $\mathrm{Fe}(\mathrm{r}=-0.83)$.

\section{Table 8}

Composition of high-purity platinum by SEM-EDS, wt.\%.

\begin{tabular}{cccccccccc}
\hline $\begin{array}{c}\text { Anal. } \\
\text { No. }\end{array}$ & $\mathrm{Fe}$ & $\mathrm{Cu}$ & $\mathrm{Ru}$ & $\mathrm{Rh}$ & $\mathrm{Ir}$ & $\mathrm{Pt}$ & Total* & Total+** & Figure \\
\hline 1 & 0.67 & 0.41 & 0.53 & & & 92.12 & 93.72 & 93.72 & \\
$2 *$ & 0.64 & 0.47 & & & & 90.65 & 91.76 & 95.98 & $3 \mathrm{a}$ \\
$3 *$ & 0.68 & 0.54 & & & & 92.42 & 93.64 & 94.35 & \\
\hline 4 & 0.46 & & & & 1.75 & 92.98 & 95.19 & 95.19 & \\
$5 *$ & 0.69 & 0.52 & 0.57 & 0.6 & & 86.54 & 88.92 & 92.11 & $3 \mathrm{I}$ \\
$6 *$ & 0.78 & & 0.54 & 0.7 & 1.48 & 83.33 & 86.83 & 89.47 & \\
$7 *$ & 0.55 & & & & & 90.78 & 91.33 & 91.98 &
\end{tabular}




\begin{tabular}{|c|c|c|c|c|c|c|c|}
\hline $8 *$ & 0.67 & 0.31 & & 87.21 & 88.19 & 89.42 & \\
\hline $9 *$ & 0.44 & & & 90.36 & 90.8 & 93.2 & \\
\hline $10 *$ & 0.54 & & & 90.82 & 91.36 & 92.17 & \\
\hline $11 *$ & 0.37 & 0.66 & & 97.96 & 98.99 & 101.43 & \\
\hline $12 *$ & 0.68 & 0.61 & & 97.35 & 98.64 & 107.12 & \\
\hline $13 *$ & 0.56 & 0.6 & & 95.37 & 96.53 & 100.59 & 3 II \\
\hline $14 *$ & 0.64 & 0.29 & & 93.43 & 94.36 & 103.99 & \\
\hline $15 *$ & 0.74 & & 1.54 & 87.1 & 89.38 & 99.85 & \\
\hline
\end{tabular}

Note: * - O, Al, Si, Mg, Ti, and Mn were not included in the calculation of the sum; **- O, Al, Si, $\mathrm{Mg}, \mathrm{Ti}$, and $\mathrm{Mn}$ included in the calculation of the sum.

\section{Discussion}

The heterogeneity of the internal texture and the complex mineral composition of the grain containing the inclusion of high-purity platinum reflect a complexity and long history of its formation and transformation. The Os-Ir-Ru lamellas, the decomposition products of the solid solution, and the oval nodule inclusions of chromite, native osmium, and olivine appeared first in the $\mathrm{Cu}$-rich isoferroplatinum. The metasomatic transformation of $\mathrm{Cu}$-rich isoferroplatinum $\left(\mathrm{Pt}_{3}\left(\mathrm{Fe}_{0.6} \mathrm{Cu}_{0.4}\right)\right)$ into the $\mathrm{Cu}-\mathrm{Pt}$ alloy $\left(\mathrm{Pt}_{1.4} \mathrm{Cu}_{0.6}\right)$, and subsequent deposition of hongshiite $\left(\mathrm{Pt}_{1.2} \mathrm{Cu}_{0.8}\right)$ was established. It should be noted that the mineralogy of the natural $\mathrm{Pt}-\mathrm{Cu}$ system still needs more detailed investigation. For example, Cabral et al. $(2014,2016)$ discovered recently new mineral kitagohaite $\left(\mathrm{Pt}_{7} \mathrm{Cu}\right)$ and $\mathrm{Pt}_{3} \mathrm{Cu}$ intermetallic compound. However, the composition of minerals in the Pt-Cu-Fe system falling in line with $\mathrm{Pt}_{3} \mathrm{Fe}-\mathrm{PtCu}$, with some miscibility gap was described previously (Tolstykh et al., 1996). The following, arsenic stage, expressed in the formation of mainly rhodarsenide and also sperrylite, irarsite and platarsite, was also quite widespread.

Hongshiite and rhodarsenide were developed not only in $\mathrm{Pt}_{3}\left(\mathrm{Fe}_{0.6} \mathrm{Cu}_{0.4}\right)$ but, also, deposited on the walls of the nodules (Fig. 3b, c). Hematite was associated with hongshiite (Fig. 3b) and, presumably, formed during the final stage. Rhodarsenide formed inclusions in hongshiite and, in addition, crystallized as an emulsion impregnation in $\mathrm{Cu}$-rich isoferroplatinum. The Os-Ir- $\mathrm{Ru}$ alloy mainly associates with $\mathrm{Cu}$-rich isoferroplatinum, $\mathrm{Cu}-\mathrm{Pt}$ alloy and to a lesser extent with rhodarsenide. The Os-Ir-Ru alloys in all $\mathrm{Cu}-\mathrm{Pt}$ mineral phases are relict and inheriting, in contrast to missing isoferroplatinum, where the Os-Ir-Ru lamellas were a result of decomposition of the solid solution. This is confirmed by the constancy of the lamella composition - Os $0.4 \mathrm{Ir}_{0.4} \mathrm{Ru}_{0.2}$ (Fig. 4C). The close association of high-purity platinum with hongshiite (Fig. 3 II) suggests a hypogene origin. The colloform-layered texture of high-purity platinum was most likely formed as a result of the coagulation of platinum colloids (Betekhtin et al., 1958; Isaenko, 1983). A colloform-layered 
texture is a "texture ... caused by sequence of layers which were deposited sequentially from colloidal solutions" (Petrov, 2012).

A possibility of platinum migration in hydrothermal solutions was considered by many researchers and confirmed by its occurrence in quartz veins (Vysotsky, 1933; Betekhtin, 1935; Ramdor, 1962; Boudreau et al., 1986; Mountain and Wood, 1988; Nixon et al., 1990; Distler and others, 2000). Cu-rich isoferroplatinum could be a source of Fe and Pt. In this case, the formation time of platinum links to the transition stage of $\mathrm{Cu}$-rich isoferroplatinum into the $\mathrm{Cu}-\mathrm{Pt}$ alloy. The removal of $\mathrm{Cu}$ and $\mathrm{Fe}$ from $\mathrm{Pt}-\mathrm{Cu}-\mathrm{Fe}$ alloys and the redeposition of pure platinum are possible processes. It also can be assumed that platinum might be present in the fluid in the form of chloride complexes (e.g., chloride complexes of $\mathrm{PtCl}_{6}{ }^{2-}$, and/or $\mathrm{PtCl}_{4}{ }^{2-}$, and/or $\mathrm{Pt}\left(\mathrm{NH}_{3}\right) \mathrm{Cl}_{6}{ }^{-}$, and/or $\operatorname{Pt}\left(\mathrm{NH}_{3}\right) \mathrm{Cl}_{5}{ }^{-}$, and/or $\mathrm{Pt}\left(\mathrm{NH}_{3}\right) \mathrm{Cl}_{3}{ }^{-}$) (Pauling, 1974; Plyusnina et al., 1995; Reith et al., 2014) which were then converted into native platinum. Further research is required in order to identify a mechanism of formation of high-purity platinum in this system. This process is not yet fully understood.

The conditions of formation of high-purity platinum differ significantly from those described in the literature: 1) thermal-metamorphogenic; and 2) hypergenic-biogenic. According to the first option (Evstigneeva et al., 1990; Tolstykh and Krivenko, 1998), the formation of high-purity platinum takes place gradually due to decomposition of sperrylite at a temperature of $900^{\circ} \mathrm{C}$ and higher. This platinum has a very high purity (998-999\%), with rhodium up to 0.5 wt.\%. Transition of sperrylite to native platinum was also confirmed experimentally (Vuorelainen and Törnroos, 1986; Evstigneeva et al., 1990). The second, supergene-biogene option, was proposed for association of aggregates of high-purity $\mathrm{Pt}$ and the $\mathrm{Pt}-\mathrm{Pd}$ alloys $\left(\mathrm{Pt}_{0.7} \mathrm{Pd}_{0.3}-\mathrm{Pt}_{0.3} \mathrm{Pd}_{0.7}\right)$ with a botryoidal, reniform, rod-shaped, and dendritic forms found in alluvial placer deposit of Minas Gerais in Brazil (Cabral et al., 2006, 2009, 2011; Cassedane et al., 1992; Fleet et al., 2002; Angeli, 2005). The native platinum aggregate size is up to $1 \mathrm{~mm}$ with a colloform and spongy internal texture. $\mathrm{Pd}, \mathrm{Hg}$ and $\mathrm{Se}$ are common in native platinum, and $\mathrm{Cu}$ and $\mathrm{Au}$ are less common. According to Cabral et al. (2006), the composition of high-purity platinum varies within $\mathrm{Pt}_{0.921-0.972} \mathrm{Pd}_{0.006}$ ${ }_{0.018} \mathrm{Hg} 0.014-0.035 \mathrm{Au}_{0-0.017} \mathrm{Cu}_{0.007-0.01} \mathrm{Se}_{0.001-0.001}$. Also, the purest platinum occurs in the marginal part of grains.

High-purity platinum could be formed in other geological settings. Platinum with palladium and rhodium (Table 9) was recorded in pentlandite and pyrrhotite in the pyroxenite ore of the Merensky Reef at the Rustenburg Platinum Mine (Kingston and El-Dosuky, 1982). Its formation was associated with the extraction of platinum from the melt by ascending fluid flows during the sulfurisation of silicate melts (Marakushev et al., 2004). The genesis of platinum remains unclear (Table 8) in chromitite and metamorphosed dunites of the Ulor ultramafic massif, Western 
Sangilen, Tuva (Agafonov et al., 2000). The submicroscopic grains of $\mathrm{Pt}_{0.96} \mathrm{Fe}_{0.04}$ and sponge submicroscopic aggregates of high-quality platinum were identified (Table 9) in black shales of the Kursk Magnetic Anomaly (KMA) (Rudashevsky et al., 1995). The formation of PGM and highpurity platinum took place under the influence of the following factors: sedimentary-chemogenic and biogenic; fluid-magmatic; and metamorphic with extraction of ore matter from protoliths and its mobilization during the functioning of deep focal zones and hydrothermal processes (Chernyshev, 2009).

\section{Table 9}

Element composition of high-purity Pt, wt.\%.

\begin{tabular}{cccccccccc}
\hline Object & $\mathrm{Pt}$ & $\mathrm{Pd}$ & $\mathrm{Fe}$ & $\mathrm{Cu}$ & $\mathrm{Os}$ & $\mathrm{Ru}$ & $\mathrm{Rh}$ & Cумma & Reference \\
\hline 1 & 99.3 & 0.3 & & & & & 0.2 & 99.8 & $\begin{array}{c}\text { Kingston et al. } \\
(1982)\end{array}$ \\
\hline 2 & 99.49 & & 0.02 & 0.07 & 0.07 & 0.03 & 0.15 & $99.83 *$ & Agafonov et al. \\
& 98.22 & & 0.05 & 0.10 & 0.03 & 0.01 & 0.15 & $98.46 *$ & $(2000)$ \\
\hline 3 & 99.1 & & 1.1 & & & & & 100.2 & Rudashevsky et al. \\
& 100.1 & & & & & & & 100.1 & $(1995)$ \\
4 & 99.59 & & 0.07 & 0.1 & & & 0.23 & 100.02 & Tolstykh, Krivenko \\
& 99.36 & & & 0.08 & & & 0.46 & 99.90 & $(1998)$ \\
\hline
\end{tabular}

Notes: 1 - Merensky Reef at the Rustenburg Platinum Mine, 2 - Ulorsk hyperbasite massif, Western Sangilen, Tuva, 3 - black shales of KMA; * - Ir, As, S, Ni were below detection limits; 4 Burguli River of Stanovaya folded region.

\section{Conclusions}

1. High-purity platinum occurs in microinclusions of the colloform-layered texture in the Pt-Fe$\mathrm{Cu}$ grain. The thickness of microinclusions is several microns, and the thickness of its constituent microlayers is $50-100$ nanometers. The only admixture in high-purity platinum is iron $(0.37$ to 0.78 wt.\%). The main minerals of the PGM grain are $\mathrm{Cu}$-rich isoferroplatinum, copper platinum, hongshiite and rhodarsenide.

2. The most important feature of the PGM grain with high-purity platinum is its significant transformation. The initial mineral form of this PGM was probably isoferroplatinum that was completely replaced. Isoferroplatinum contained the lamellas of rutheniridosmine and nodule inclusions of various compositions, including osmium, chromite and olivine. The transformation took place in the already deformed grain, broken by microcracks, during two stages: 1 - copper stage, including three substages ( $\mathrm{Cu}$-rich isoferroplatinum, copper platinum and hongshiite); and 2 - 
arsenic stage (rhodarsenide). These stages might have occurred during a long geological time, in various lithogenetic and geodynamic settings.

3. The lamellae of Ru-Os-Ir alloy and the inclusion of osmium were preserved unchanged during the metasomatic replacement of isoferroplatinum by $\mathrm{Cu}$-rich isoferroplatinum and $\mathrm{Cu}-\mathrm{Pt}$ alloys.

4. The formation of high-purity platinum was separated in time from the formation of $\mathrm{Cu}$-rich isoferroplatinum and originated from postmagmatic fluids.

\section{Acknowledgements}

The authors gratefully acknowledge the valuable assistance of Ludviga Boboshko for help in the paper preparation; Dr. Nikolay Karmanov and Michael Khlestov for support with SEM/EDS analysis; Dr. Vladimir Korolyuk for his help with microprobe analysis. Special thanks are due to Professor Franco Pirajno, Dr. Alexander Yakubchuk, and anonymous reviewer for comments, questions and careful and helpful review.

This work was supported financially by the Russian Foundation for Basic Research (grant 15-0506950).

\section{References}

Agafonov, L.V., Kuzheget, K.S., Oidup, I.K., Mongush, A.A., 2000. Solid ore deposits of platinum-group minerals in ultrabasic and basic rocks of Tuva. Geology and mineral resources of Siberia. Krasnoyarsk, KNIIGiMS 1, 167-171.

Agafonov, L.V., Kuzheget, K.S., Oydup, I.K., Stupakov, C.I., 1992. Platinum-group elements and other native elements of Tuva. Dokl. Akad. Nauk 327(3), 379-383.

Agafonov, L.V., Velinskii, V.V., Loskutov, I.Yu., 1996. Unusual mineral assemblage of the noble metals in the dikes within Togul-Sungai Ultramafic Massif, Salair Ridge. Dokl. Akad. Nauk 351(4), 505-508.

Betehtin, A.G., 1935. Platinum and Other Minerals of Platinum-group Elements. AS USSR, Moscow, 148 pp., [in Russian].

Betekhtin, A.G., Genkin, A.D., Filimonova, F.F., Shadlun, I.E., 1958. Ore Textures and Structures. Gosgeoltekhizdat, Moscow, [in Russian].

Angeli, N., 2005. Platinum group minerals in eastern Brazil. Platinum Metals Rev. 49(1), 41-53.

Boudreau, A.E., Mathez, E.A., McCallum, I.S., 1986. Halogen geochemistry of the Stillwater and Bushveld complexes: Evidence for transport of the platinum-group elements by Cr-rich fluids. Journ. Petrology 27, 967-986. 
Cabral, A.R., Beudoin, G., Kwitko-Ribeiro, R., Lehmann, B., Polonia, J.C., Choquette, M., 2006. Platinum-palladium nuggets and mercury-rich Palladiferous Platinum from Serro, Minas Gerais, Brazil. The Canadian Mineralogist 44, 385-597.

Cabral, A.R., Lehmann, B., Jedwab, J., 2016. A platinum-copper nugget rich in calcite: evidence for hydrothermal $\mathrm{Pt}_{3} \mathrm{Cu}$ ). Canadian Mineralogist 54, 401-407.

Cabral, A.R., Lehmann, B., Tupinamba, M., Schlosser, S., Kwitko-Ribeiro, R., de Abreu, F.R., 2009. The planitiferous Au-Pd belt of Minas Gerais, Brazil and genesis of its botryoidal Pt-Pd-Hg aggregates. Econom. Geol. 104, 1265-1276.

Cabral, A.R., Radtke, M., Munnik, F., Lehmann, B., Reinholz, U., Riesemeier, H., Tupinamba, M., Kwittko-Ribeiro, R., 2011. Iodine in alluvial platinum-palladium nuggets: Evidence for biogenic precious-metal fixation. Chemical Geology 281, 125-132.

Cabral, A.R., Skála, R., Vymazalova, A., Kallistova, A., Lehmann, B., Jedwab, J., Sidorinová, T., 2014. Kitagohaite, $\mathrm{Pt}_{7} \mathrm{Cu}$, a new mineral from the Lubero region, North Kivu, Democratic Republic of the Congo. Mineralogical Magazine 78(3), 739-745.

Cabri, L.J., Harris, D.C., Weiser, T.W., 1996. Mineralogy and distribution of Platinum-group mineral (PGM) in placer deposits. Explor. Mining Geol. 5(2), 73-167.

Cassedane, J.P. and Alves, J.N., 1992. Palladium and platinum from Córrego Bom Sucesso, Minas Gerais. Brazil. Mineral. Rec. 23, 471.

Chernyshev, N.M., 2009. Minerals of noble metals in the ore of the black shale type of the Voronezh crystalline massif (Central Russia). Notes of the Russian Mineralogical Society 38(1), 3650.

Distler, V.V., Yudovskaya, M.A., Prokof'ev, V.Yu., Sluzhenikin, S.F., Mokhov, A.V., Mun, Ya.V., 2000. Hydrothermal Platinum mineralization of the Waterberg deposit (Transvaal, South Africa). Geol. Ore Deposits 42(4), 328-339.

Evstigneeva, T.L., Kim, A.A., Nekrasov, Ya.V., 1990. Dearsenization of sperrylite in the environment. Mineral. Journal 12(3), 90-96.

Fleet, M.E., de Almeida, C.M., Angeli, N., 2002. Botryoidal platinum, palladium and potarite from the Bom Sucesso stream, Minas Gerais, Brazil: compositional zoning and origin. Can. Mineralog. 40, 341-355.

Gornostayev, S., Crocket, J., Mochalov, A.G., Laajoki, K.V.O., 1999. The platinum-group minerals of the Baimka placer Deposits, Aluchin Horst, Russian Far East. Can. Mineral. 37, 11171129.

Gusev, A.I., Grinev, R.O., Chernyshev, A.I., 2004. Petrology and ore potential of the Seglebir ophiolite association (north-eastern Gorny Altai and southern Gornaya Shoria), in: Petrology of Magmatic and Metamorphic Complexes. Proc. Conf. TsNTI, Tomsk, Issue 4, 130-133. 
Isaenko, M.P., 1983. The identification guide of textures and structures of ores. Moscow: Nedra. Kingston, G.A., El-Dosuky, B.T., 1982. A contribution on the Platinum-group mineralogy of the Merensky Reef at the Rustenburg Platinum Mine. Economic Geology 77, 1367-1384.

Krivenko, A.P., Tolstykh, N.D., Nesterenko, G.V., Lazareva, E.V., 1994. Types of mineral associations of platinoids in auriferous placers of the Altai-Sayan region. Russian Geology and Geophysics 35(1), 58-65.

Lavrent'ev, Yu.G. and Usova, L.V., 1994. New version of the Karat program for quantitative Xray spectral microanalysis. J. Anal. Chem. 49(5), 465-468.

Marakushev, A.A., Paneyakh, N.A., Gorbachev, N.S., Zotov, I.A., 2004. Mineral-geochemical specifics of giant deposits of chromium and platinum metals and the problem of the depth of their mantle sources. In: Large and Super-large Deposits: Location Patterns and Conditions of formation / Ed. D.V. Rundquist. Moscow: IGEM RAS, [in Russian].

Mountain, B.W., Wood, S.A., 1988. Chemical controls on the solubility, transport and deposition of platinum and palladium in hydrothermal solutions: A thermodynamic approach. Econom. Geology 83, 492-510.

Nixon, G.T., Cabri, L.J. \& Laflamme, J.H.G., 1990. Platinum-group element mineralization in lode and placer deposits associated with the Tulameen Alaskan-type complex, British Columbia. Can. Mineral. 28, 503-535.

Okrugin, A.V., 2000. Placer Platinum of the Siberian Platform. Yakutsk: YaP Publishing House of SB RAS, [in Russian].

Pauling, L., 1974. General Chemistry. Moscow: Mir, [in Russian].

Petrov O.V. (Ed.), 2012. Geological Glossary. The third edition. T. 3. R-Ya. Saint Petersburg: Publishing house VSEGEI.

Podlipsky, M.Yu., Krivenko, A.P., 2001. New data on geological structure, lithology, and formational type of the Kaigadat massif as a primary source of Pt- and Fe-bearing PGM in placers, in: Topical Problems of Geology and Mineralogy of Southern Siberia. Proc. Conf., October 31November 2, Elan', Kemerovo region, Novosibirsk: 126-132, [in Russian].

Podlipsky, M.Yu., Nesterenko, G.V., Krivenko, A.P., 2007. Isoferroplatinum mineral assemblage in the northwestern Salair gold plaser. Russian Geology and Geophysics 48(3), 291298.

Plyusnina, L.P., Nekrasov, I.Ya., Scheka, Zh.A., 1995. Experimental studies of the solubility of platinum in water-chloride solutions at $300-500^{\circ} \mathrm{C}$ and $1 \mathrm{kbar}$. Dokl. RAS 340(4), 525-527, [in Russian].

Ramdorh, P., 1962. Ore Minerals and their Intergrowths. Izd. Inostr. Lit., Moscow, [in Russian]. 
Reith, F., Campbell, S.G., Ball, A.S., Pring, A., Southan, G., 2014. Platinum in Earth surface environments. Earth-Science Reviews 131, 1-21.

Rudashevsky, I.S., Knauf, V.V., Chernyshev, N.M., 1995. Platinum-group minerals from the black shales of the KMA. Doklady Earth Science 344(1), 91-95.

Tolstykh, N.D., Krivenko, A.P., 1998. Native high-purity platinum, its origin, and the nomenclature of Pt-Fe alloys. Doklady Earth Science 361A, 775-777.

Tolstykh, N.D., Krivenko, A.P., Baturin, S.G., 1996. Compositional features of native platinum from different minerals assemblages of platinum-group elements. Russian Geology and Geophysics 37(3), 35-43.

Tolstykh, N., Krivenko, A., Sidorov, E., Laajoki, K., Podlipsky, M., 2002. Ore mineralogy of PGM placers in Siberia and the Russian Far East. Ore Geol. Rev. 20, 1-25.

Tolstykh, N., Kozlov, A., Telegin, Y., 2015. Platinum mineralization of the Svetly Bor and Nizhny Tagil intrusions, Ural Platinum Belt. Ore Geol. Rev. 67, 234-243.

Tolstykh, N.D., Lapukhov, A.S., Krivenko, A.P., Lazareva, E.V., 1999. Platinum-group minerals in gold placers in northwestern Salair. Russian Geology and Geophysics 40(6), 900-910.

Vuorelaien, Y., Törnroos, R., 1986. Man-made Pt-PtAs2 spherules after sperrylite from alluvial Deposits in Finnish Lapland. Can. Mineral 24, 523-528.

Voronova L.I., Polyakova V.P., Savitskii E.M.,1984. Alloys of the system Pt-Os. Russ. Metall. 5, 201-203.

Vysotsky, N.K., 1933. Platinum and Areas of Its Production. Part V. Overview of Platinum Deposits outside the Urals. Izd. AN SSSR, Leningrad, [in Russian].

Winkler O., 1943. Untersuchungen uber die Abriebfestigkeit von Edelmetallen und hochschmelzenden Unedelmellen. Z. Elektrochem. 49, 221-228.

Zhmodik, S.M., Agafonov, L.V., Nesterenko, G.V., Kolpakov, V.V., Shcherbakov, Yu.G., 2004. Au-PGE placer mineralization in northern Kuznetsk Alatau, in: Gold of Siberia and Russian Far East: Geology, Geochemistry, Technology, Economy, Ecology. Ulan-Ude: 81-84, [in Russian].

Zaykov, V.V., Melekestseva, I.Yu., Zaykova, E.V., Kotlyarov, V.A., Kraynev, Y.D., 2017. Gold and platinum group minerals in placers of the South Urals: Composition, microinclusions of ore minerals and primary sources. Ore Geol. Rev. 85, 299-320.

Zhmodik, S.M., Nesterenko, G.V., Airiyants, E.V., Belyanin, D.K., Kolpakov, V.V., Podlipsky, M.Yu., Karmanov, N.S., 2016. Alluvial platinum-group minerals as indicators of primary PGE mineralization (placers of southern Siberia). Russian Geology and Geophysics 57, 1437-1464. 